\title{
Disruption of ST5 is associated with mental retardation and multiple congenital anomalies
}

\author{
Ina Göhring, ${ }^{1}$ Andreas Tagariello, ${ }^{1}$ Sabine Endele, ${ }^{1}$ Claus C Stolt, ${ }^{2}$ Michella Ghassibé, ${ }^{3}$ \\ Malcolm Fisher, ${ }^{4}$ Christian T Thiel, ${ }^{1}$ Udo Trautmann, ${ }^{1}$ Miikka Vikkula, ${ }^{3}$ \\ Andreas Winterpacht, ${ }^{1}$ David R FitzPatrick, ${ }^{4}$ Anita Rauch ${ }^{1,5}$
}

${ }^{1}$ Institute of Human Genetics, Friedrich-Alexander University Erlangen-Nuremberg, Erlangen, Germany ${ }^{2}$ Institute of

Biochemistry, Emil-Fischer Centre, Friedrich-Alexander University Erlangen-Nuremberg, Erlangen, Germany ${ }^{3}$ de Duve Institute, Université catholique de Louvain, Brussels, Belgium ${ }^{4}$ Medical and Developmental Genetics Section, MRC Human Genetics Unit, Western General Hospital, Edinburgh, UK ${ }^{5}$ Institute of Medical Genetics, University of Zurich, Schwerzenbach-Zurich, Switzerland

\section{Correspondence to}

Professor Anita Rauch, Institute of Medical Genetics, University of Zurich, Schorenstrasse 16, CH-8603

Schwerzenbach-Zurich, Switzerland; anita.rauch@medgen.uzh.ch

Received 26 May 2009 Revised 10 September 2009 Accepted 10 September 2009 Published Online First 19 October 2009

\begin{abstract}
Background The authors observed a patient with a cryptic subtelomeric de novo balanced translocation 46 , $X Y$.ish $t(11 ; 20)(p 15.4 ; q 13.2)$ presenting with severe mental retardation, muscular hypotonia, seizures, bilateral sensorineural hearing loss, submucous cleft palate, persistent ductus Botalli, unilateral cystic kidney dysplasia and frequent infections.

Methods and Results Fluorescence in situ hybridisation mapping and sequencing of the translocation breakpoints showed that no known genes are disrupted at 20q13.2 and that ST5 (suppression of tumorigenicity 5; MIM 140750 ) is disrupted on 11p15.4. By quantitative PCR from different human tissues, the authors found ST5 to be relatively evenly expressed in fetal tissues. ST5 expression was more pronounced in adult brain, kidney and muscle than in the corresponding fetal tissues, whereas expression in other tissues was generally lower than in the fetal tissue. Using RNA in situ hybridisation in mouse, the authors found that St5 is expressed in the frontal cortex during embryonic development. In adult mouse brain, expression of $S t 5$ was especially high in the hippocampal area and cerebellum.

Conclusion Hence, the authors suppose that ST5 plays an important role in central nervous system development probably due to disturbance of DENN-domain-mediated vesicle formation and neurotransmitter trafficking. Thus, these findings implicate ST5 in the aetiology of mental retardation, seizures and multiple congenital anomalies.
\end{abstract}

\section{INTRODUCTION}

Mental retardation (MR) is defined as a significant impairment of cognitive and adaptive functions with onset before age 18 years and affects about $2-3 \%$ of the population. It occurs as an isolated trait or as part of many syndromes, and, despite significant improvement in the identification of underlying causes, the majority of cases currently remain without definitive diagnosis. ${ }^{1}$ Since MR is extremely heterogeneous and occurs sporadically in most patients, identification of novel causative genes by linkage analysis is difficult. In such cases, balanced chromosomal rearrangements, which occur in $0.6 \%$ of mentally retarded patients, are one of the most promising sources for targeting particular genomic segments or genes responsible for MR or other developmental disorders in humans. ${ }^{1}$ The phenotypic effects observed in patients with truly balanced chromosomal rearrangements can be explained by the disruption of genes or by alteration of expression of genes residing at the chromosomal breakpoints or in close proximity to them. In this study, breakpoint mapping of a balanced de novo translocation $t(11 ; 20)(p 15.4 ; q 13.2)$ in a patient with $M R$, seizures and multiple congenital anomalies led to the identification of ST5 (suppression of tumorigenicity 5; MIM 140750) as a novel gene for syndromic MR.

\section{METHODS \\ Fluorescence in situ hybridisation (FISH)}

Region-specific bacterial artificial chromosome (BAC) clones for FISH mapping were selected from the NCBI and UCSC genome browsers (http:// www.ncbi.nlm.nih.gov, http:/genome.ucsc.edu/). FISH analyses were performed using metaphase chromosomes prepared from lymphoblastoid cell lines. Genomic BAC DNAs were fluorescently labelled with Cy3-dCTP (Amersham Biosciences, Freiberg, Germany) by standard nick translation with the DIG-Nick-Translation Kit (Roche, Basel, Switzerland). The probes were blocked with Cot-1 DNA to suppress repetitive sequences. Metaphase spreads were hybridised at $37^{\circ} \mathrm{C}$ overnight with the Cy3-labelled BAC probes and a specific FluoroX (Amersham Biosciences)-labelled subtelomeric control probe (PAC probes GS-11q770-G7 or GS20p1061-L1). ${ }^{2}$ After post-hybridisation washes, chromosomes were counterstained with 4',6diamidino-2-phenylindole (DAPI; Serva, Heidelberg, Germany). Images were captured on a Zeiss Axioplan 2 microscope with a CCD camera and processed with the Isis Software (Metasystems. Altlussheim, Germany).

\section{Long-template PCR and mini-FISH analyses}

All long-template PCRs were performed with the Expand Long Template PCR System (Roche Diagnostics, Basel, Switzerland) according to the recommendations of the manufacturer with primer pairs chosen from the genomic sequence of the breakpoint-spanning BAC clones. Annealing temperatures and elongation times were optimised for each primer pair. Purified long-template PCR products (OIAquick PCR Purification Kit, Qiagen, Hilden, Germany) were labelled with Cy3-dCTPs by standard nick translation as described above, and FISH was performed on metaphase spreads. Hybridisation time was elongated to $48 \mathrm{~h}$.

\section{Breakpoint sequencing}

Primer pairs for breakpoint-spanning PCRs were chosen from the genomic sequence of the breakpointcontaining segments on chromosomes 11 and 20 . 
Figure 1 Subtelomeric screening and cytogenetic analysis in our patient revealed a cryptic subtelomeric translocation with the karyotype $46, X Y$, $t(11 ; 20)(p 15.4 ; q 13.2)$. (A) Partial karyogram and ideogram. In addition, chromosome painting using libraries for chromosome 11 (green) and 20 (red) was performed (B). For mapping of the translocation breakpoints, fluorescence in situ hybridisation was performed with bacterial artificial chromosome (BAC) clones. BAC clones RP11$152 \mathrm{H} 18$ (red) on chromosome 11 (with 11 qter probe, green) (C) and RP5906P16 (red) on chromosome 20 (with 20pter probe, green) (D) showed hybridisation signals localised on both derivative chromosomes, demonstrating that they spanned the breakpoint.
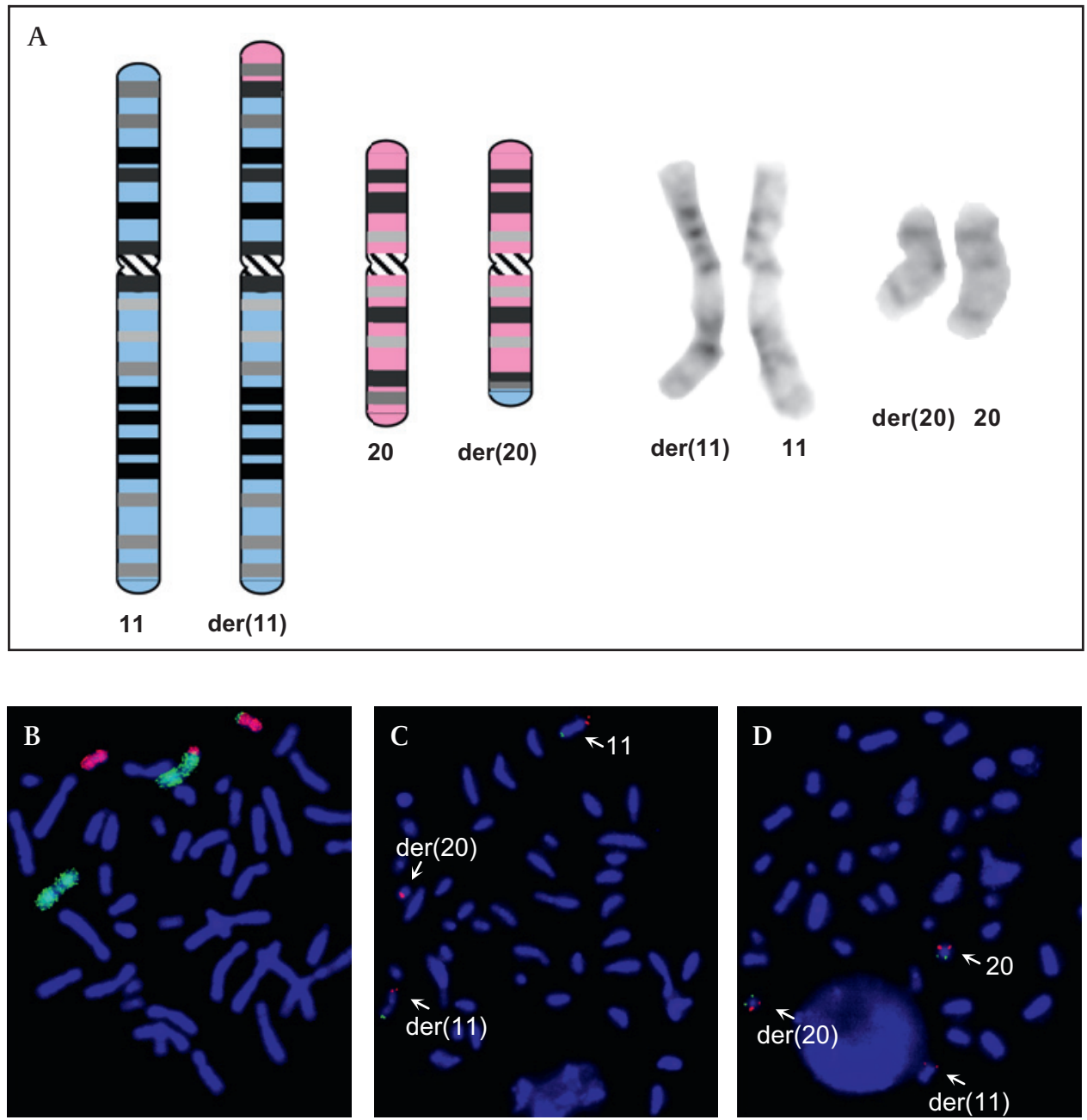

Different combinations of forward and reverse primers were used to amplify a breakpoint-spanning PCR product with the Expand $20 \mathrm{~kb}^{\text {Plus }}$ (PCR System, dNTPack; Roche Diagnostics) according to the manufacturer's recommendations. We amplified the breakpoint-spanning PCR product from the derivative chromosome 11 using the following primer pair Chr11-5'TCTTCTGCCTAATGGTCAGC-3' and Chr20-5'-CTGCAAGCCAAGAAGAGAGG-3'. The breakpoint-spanning PCR product from the derivative chromosome 20 was generated with the primer pair Chr11-5'-CCATCCATCTCTCCTCATTCCTTAAGTT-3' and Chr20-5'-CCAATTAAGATGAGAAGATCCCTCTAGGA-3'. Dye terminator cycle sequencing of the purified PCR products was carried out using the DNA Sequencing Kit BigDye-Terminator Cycle Sequencing Ready Reaction (Applied Biosystems, Forster City, CA, USA). Sequencing products were separated on an Applied Biosystems ABI3730 Sequencing System. The NCBI BLAST program (http://ncbi.nlm.nih.gov/BLAST) and the UCSC BLAT program (http://genome.ucsc.edu/cgi-bin/ hgBlat) were used to analyse the sequencing files.

\section{Copy-number analysis}

To investigate the presence of the reported ST5 copy-number variations in well-characterised control individuals, we performed long-range PCR using intronic primers from intron 7 to intron 10, which generated a $3.4 \mathrm{~kb}$ product in 192 healthy blood donors. Copy-number changes larger than $100 \mathrm{~kb}$ were investigated in 667 mentally healthy individuals using data generated with the Affymetrix GeneChip SNP array 6.0 and the
Genotyping Console 3.0.2 software to call segments of copynumber changes.

\section{Quantification of ST5 expression by real time reverse transcriptase (RT)-PCR}

cDNAs were taken from the Human Fetal Multiple Tissue cDNA (MTC) Panel and from the Human Adult Multiple Tissue cDNA (MTC) Panel (Clontech, Mountain View, CA, USA). RT-PCR studies were performed as described previously using the ST5 predesigned TaqMan Gene Expression Assay (Applied Biosystems; Hs00373572_m1), and normalised against the mean of four endogenous controls ( $\beta 2$-microglobulin, $\beta$-actin, hypoxanthine phosphoribosyltransferase 1 and RNA polymerase II). ${ }^{3}$ Relative expression levels were compared with the mean value of STS expression in fetal brain.

\section{RNA in situ hybridisation}

Murine expression of $S_{t} 5$ was investigated on embryonic days 8.5, 13.5 and 15 (E8.5, E13.5, E15), in brain and kidneys of newborn mice on days 6, 10 and 15 (P6, P10, P15) and in brains of 7-week-old mice and adult mice. Tissue was OCT (TissueTek, Sakura Finetek, Heppenheim, Germany) embedded and stored at $-80^{\circ} \mathrm{C}$. Serial cryostat sections at $10-12 \mu \mathrm{m}$ were fixed in $4 \%$ paraformaldehyde/phosphate-buffered saline. A 448 bp mouse St 5 riboprobe was generated by PCR using the primers ggagactgtgggtcactactcc and aatatggtgaaggcagaagtgg and subcloning of the amplicon into the pCR4-TOPO vector (Invitrogen) as recommended by the manufacturer. The probe was labelled according to the in vitro transcription kit (DIG-RNA 


\section{A}

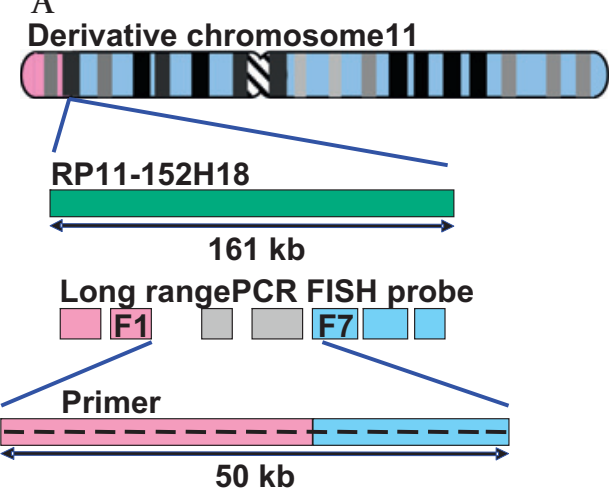

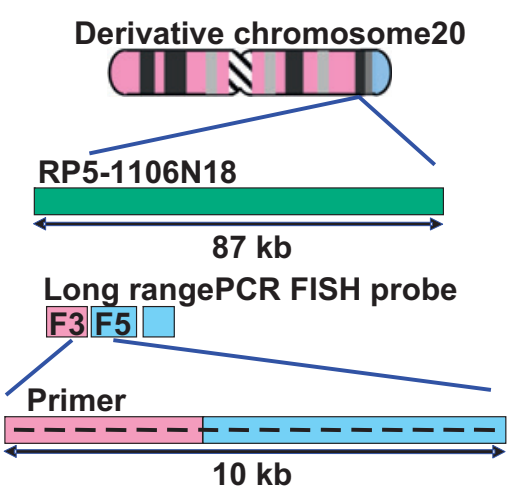

$10 \mathrm{~kb}$

\section{Chromosome 11 \\ Chromosome 20 \\ Breakpoint spanning BAC probe}

B

\section{Breakpointsequenceon der11}

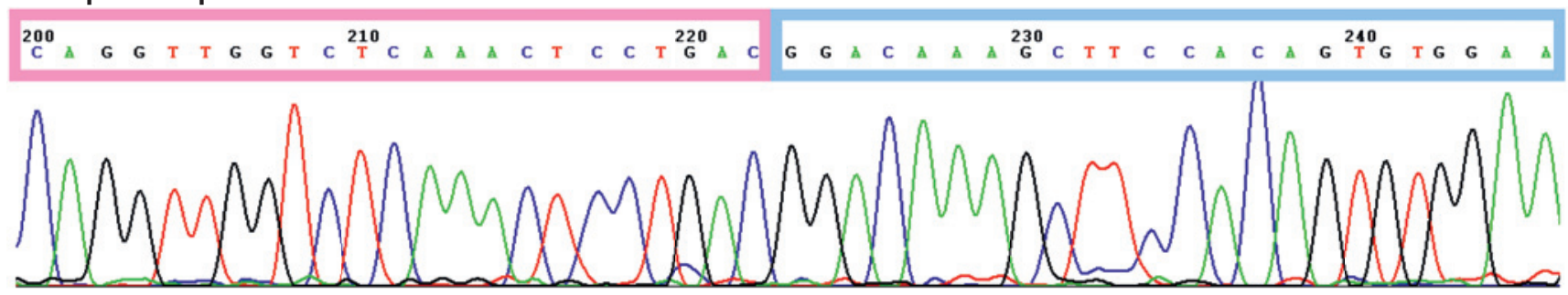

\section{Chromosome 11 sequence} Chromosome 20 sequence

\section{Breakpointsequenceon der20}

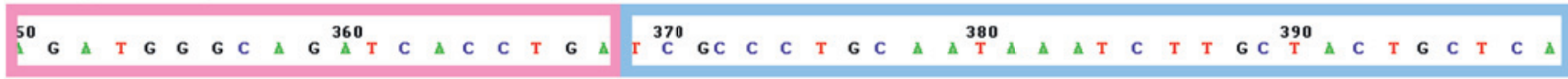

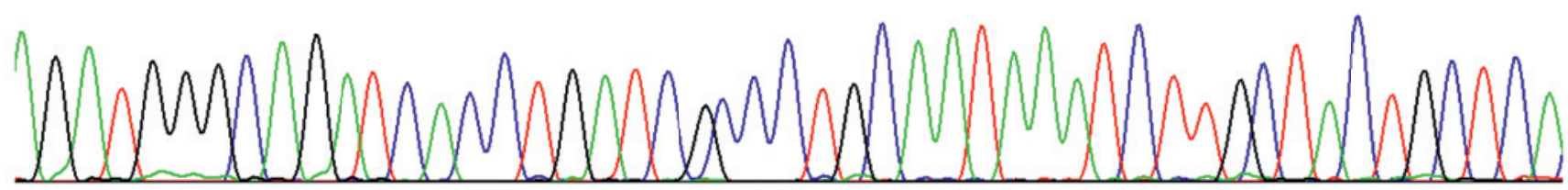

Figure 2 Fluorescence in situ hybridisation (FISH) studies narrowed the breakpoint regions to a $161 \mathrm{~kb}$ region on chromosome 11 and an $87 \mathrm{~kb}$ region on chromosome 20 (A). 'Mini-FISH' probes were created from the breakpoint-spanning bacterial artificial chromosomes (BACs) by long-template PCR. Therewith it was possible to narrow the breakpoint region on the derivative chromosome 11 to a $50 \mathrm{~kb}$ fragment between probes F1 and F7. We obtained signals on chromosome 20 with probe Chr11-F1 indicating that this probe is translocated, whereas probe Chr11-F7 was not translocated. For the grey illustrated 'mini-FISH' probes, we were not able to achieve usable results from the FISH analysis. On the derivative chromosome 20, we narrowed the breakpoint to a $10 \mathrm{~kb}$ fragment between probes Chr20-F3 and Chr20-F5. While we found probe Chr20-F3 not to be translocated, probe Chr20-F5 showed signals on chromosome 11, demonstrating that this probe is translocated. Finally, we exactly mapped the breakpoints by sequencing of breakpointspanning long-template PCR products from both derivative chromosomes (B).

Labeling Mix; Roche) using $10 \mu$ l linearised plasmid, $2 \mu 110 \times$ Labeling Mix, $2 \mu 110 \times$ transcription buffer, $1 \mu \mathrm{l}$ RNase inhibitor, $2 \mu$ polymerase (T3 or T7 according to sense or antisense probes) and $3 \mu \mathrm{l} \mathrm{PCR}$-grade water. The mix was incubated at $37^{\circ} \mathrm{C}$ for $2 \mathrm{~h}$. Serial sections were hybridised with digoxigenin (DIG-UTP)labelled RNA sense and antisense probes and with a control riboprobe (COL2A1). Hybridisation was carried out at $60^{\circ} \mathrm{C}$ in $50 \%$ formamide overnight. The slides were washed four times in $2 \times$ saline-sodium citrate (SSC), three times in $0.1 \times$ SSC (all incubations $30 \mathrm{~min}$ ) and finally $5 \mathrm{~min}$ in phosphate buffered saline (PBT). Reaction was blocked with Blocking buffer (Roche Diagnostics) for $40 \mathrm{~min}$, and then the slides were incubated with alkaline phosphatase (AP)-conjugated Anti-DIG $\mathrm{F}_{\mathrm{ab}}$ fragments (Roche Diagnostics) at $37^{\circ} \mathrm{C}$ for $1 \mathrm{~h}$. The slides were counterstained with toluidine blue (Roche Diagnostics). Images were captured on a MZ16 microscope (Leica, Wetzlar, Germany) with a CCD camera (DC300, Leica) and processed with the IM-Software 1.20 (Leica).

\section{RESULTS}

\section{Case report}

The patient described here was introduced to the genetics clinic at the age of 3.5 years, presenting with severe MR, muscular hypotonia, seizures, bilateral sensorineural hearing loss, submucous cleft palate, persistent ductus arteriosus Botalli, unilateral cystic kidney dysplasia, and a history of frequent infections. He also showed dysmorphic facial features including a high forehead, high arched, laterally placed eyebrows, broad glabella, mild hypertelorism, narrow, upslanting palpebral fissures, a small mouth with thin upper lip and everted lower lip, a broad nasal bridge and hypoplastic nasal wings. The proband was the third child of healthy, unrelated European parents. As ultrasound examinations showed unilateral cystic kidney dysplasia, prenatal cytogenetic analysis of chorionic villi specimens was performed and showed a normal karyotype. The patient was born at 38 weeks of gestation with a length of $51 \mathrm{~cm}$ (50th centile), a weight of $3490 \mathrm{~g}(50-75$ th centile) and a head circumference of 
Figure 3 The breakpoint (vertical arrow) on chromosome 11 is located within intron 4 in the $5^{\prime}$-UTR region of the ST5 gene, 725 bp before the first coding exon $5(A)$, indicating that the complete coding region of ST5 is separated from its promoter region. ST5 spans $217.600 \mathrm{bp}$ and consists of four non-coding and 19 coding exons. Three isoforms of ST5 are described, all containing a DENN-domain region 3'. Two ST5 transcripts also include an $A B L 1$-interacting region. Expression analysis of ST5 with quantitative PCR on commercially available cDNA panels showed relatively even expression in fetal tissues (B). In adult brain, kidney and muscle ST5 expression was much higher than in the corresponding fetal tissues, whereas expression in other tissues was generally lower than in the respective fetal tissue.

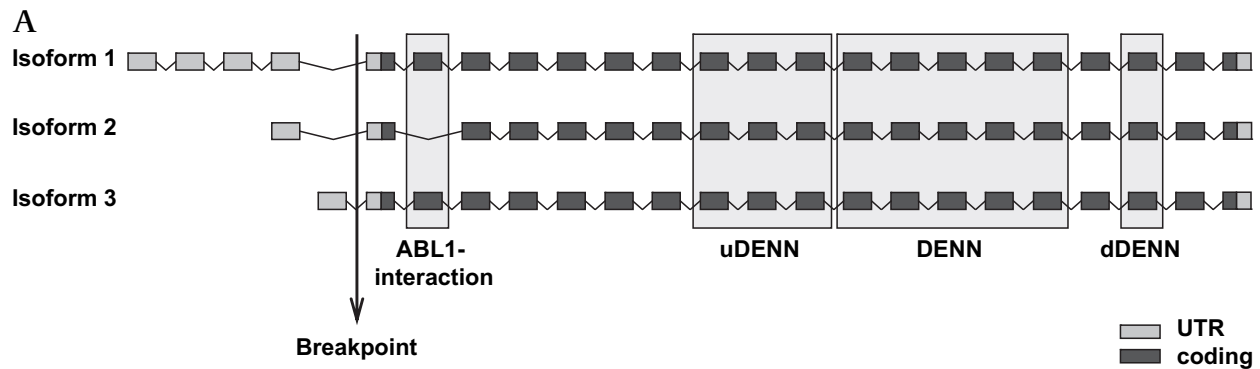

B

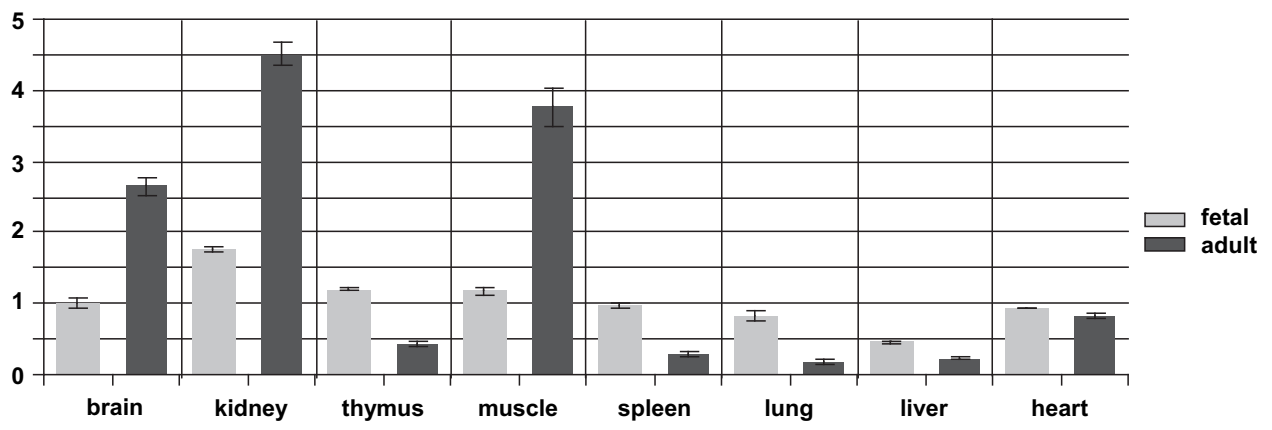

$34 \mathrm{~cm}$ (25-50th centile). He showed delayed achievement of motor milestones. At the age of 3 years and 6 months he could neither walk nor speak. At the age of 7 years, the boy was able to walk with orthoses, but still showed no speech. Absences and focal seizures with generalisation, which were resistant to therapy, were observed starting at the age of 6 months. Neither brain MRI nor metabolic testing showed any abnormalities.

Subtelomeric screening by FISH using an optimised BAC/PAC set ${ }^{2}$ showed an apparently balanced translocation, which was in combination with whole-chromosome painting and highresolution GTG banding interpreted as 46,XY.ish $t(11 ; 20)$ (p15;q13.3)(wcp20+,200TEL14+,11PTEL03-,wcp11+;wcp20+, 200TEL14-,wcp11+,11PTEL03+) (figure 1A,B). Parental karyotypes including subtelomeric FISH studies were normal. As it had recently been shown that apparently balanced translocations may be associated with microdeletions or duplications within the breakpoint region or in independent locations, ${ }^{4}$ we performed molecular karyotyping using an Affymetrix GeneChip Mapping 250K Nsp SNP array. Although seven copy-number variations were detected using the Nexus software (BioDiscovery, El Segundo, CA, USA), all of them were previously described as present in normal individuals according to the Database of Genomic Variants (http://projects.tcag.ca/variation/) and were therefore not of further interest. In particular, we revealed no significant loss or gain of copy number at either site of the breakpoints.

\section{Chromosomal breakpoint mapping}

In order to map the translocation breakpoints at a higher DNA resolution, we selected region-specific BAC clones from the databases. Individual BACs from the breakpoint regions were hybridised to the patient's metaphase spreads. On chromosome 11, BAC clone RP11-152H18 (8.6 Mb from pter) showed FISH signals localised on both derivative chromosomes, showing that it spanned the breakpoint (figure 1C). On chromosome 20, the breakpoint was spanned by the BAC clone RP5-1106N18 (49.3 $\mathrm{Mb}$ from pter) (figure $1 \mathrm{D})$. In order to narrow down the resulting breakpoint regions of $161 \mathrm{~kb}$ on chromosome 11 and $87 \mathrm{~kb}$ on chromosome 20, further breakpoint refinement was performed using 'mini-FISH' probes created by amplifying $12-18 \mathrm{~kb}$ fragments from the breakpoint-spanning BACs by long-template PCR. Amplicons were Cy3-labelled by nick translation and used for metaphase FISH. For some of the generated long-template PCR amplicons, FISH analysis did not result in usable signals, probably because of the repetitive sequences enclosed in this region. However, with this method it was possible to narrow the breakpoint region on the derivative chromosome 11 to a $50 \mathrm{~kb}$ fragment between probes Chr11-F1 and Chr11-F7 and on the derivative chromosome 20 to a $10 \mathrm{~kb}$ fragment between probes Chr20-F3 and Chr20-F5 (figure 2A). Combining the results of FISH and mini-FISH analyses, we were able to refine the breakpoint on chromosome 11 from $11 \mathrm{p} 15$ to 11 p15.4 and to change the breakpoint on chromosome 20 from $20 \mathrm{q} 13.3$ to $20 \mathrm{q} 13.2$.

We made an effort to generate breakpoint-spanning longtemplate PCR products from both derivative chromosomes. By sequencing these PCR products, we exactly mapped the breakpoint on chromosome 11 between position 8729572 and 8729575 bp (UCSC hg18) and on chromosome 20 between position 49349869 and $49349871 \mathrm{bp}$, with the loss of $3 \mathrm{bp}$ on each chromosome (figure $2 \mathrm{~B}$ ). The chromosomal break on chromosome 20 did not occur within any known or putative genes, with the closest gene residing at a distance of $91 \mathrm{~kb}$. Although the possibility of position effects on nearby genes has to be considered, so far these genes have not been associated with MR or malformations. In contrast, the breakpoint on chromosome 11 was located within intron 4 in the $5^{\prime}$-UTR region of the ST5 gene, $725 \mathrm{bp}$ before the first coding exon 5 (figure $3 \mathrm{~A}$ ). Hence we assumed that the complete coding region of this gene was separated from its promoter region.

\section{Expression analyses of ST5}

To further elucidate the involvement of ST5 in the patients' phenotype, we investigated its expression in fetal and adult human tissues by quantitative RT-PCR as well as by RNA in situ hybridisation on mouse sections. RT-PCR studies were performed using the ST5 pre-designed TaqMan Gene Expression Assay (ABI, Hs00373572_m1), which recognises exons 7 and 8 and thus detects 

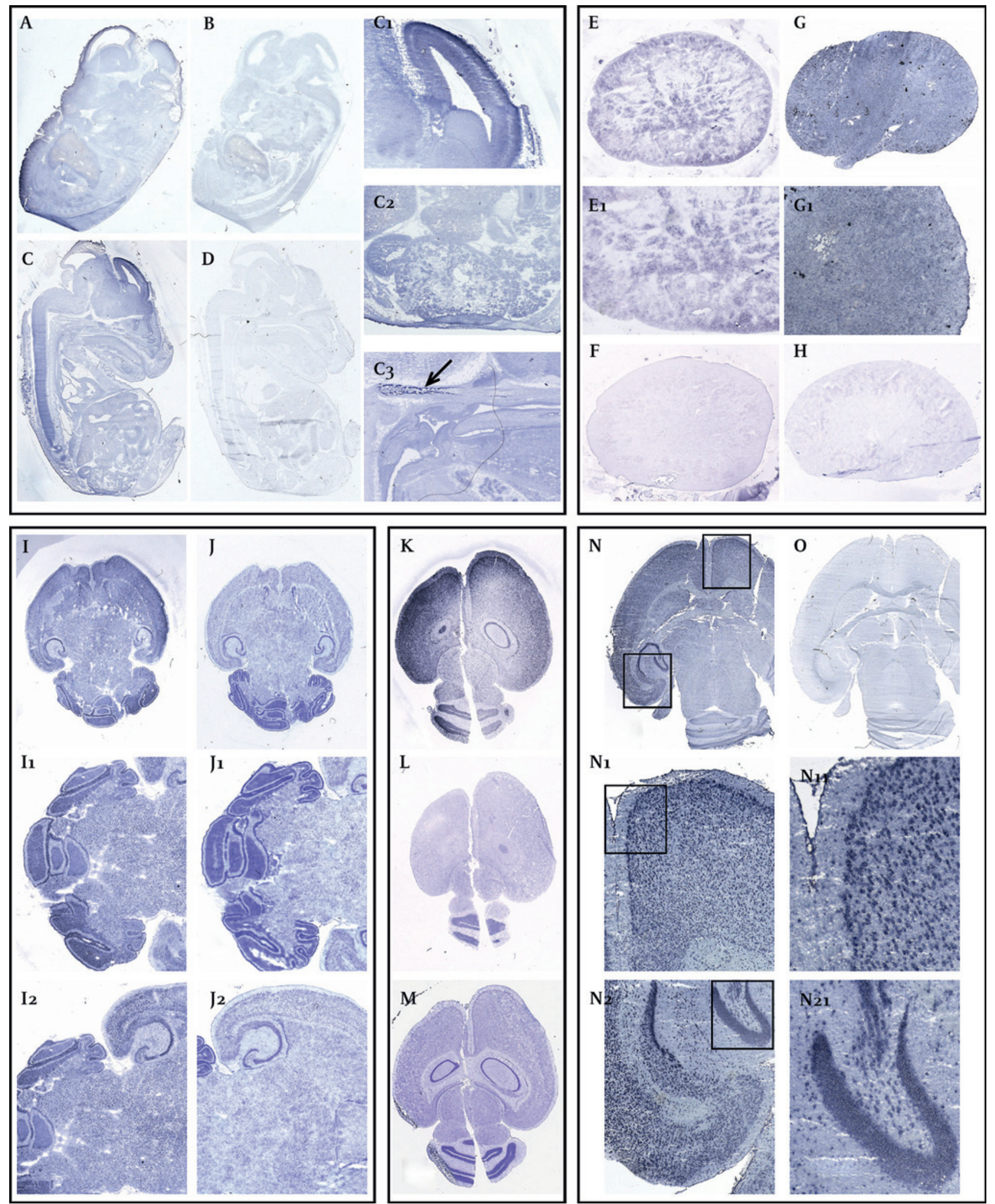

L

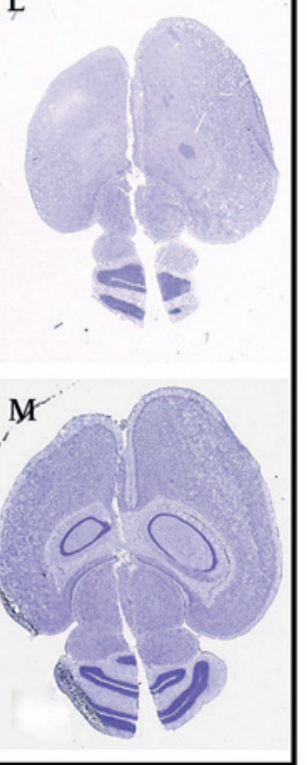

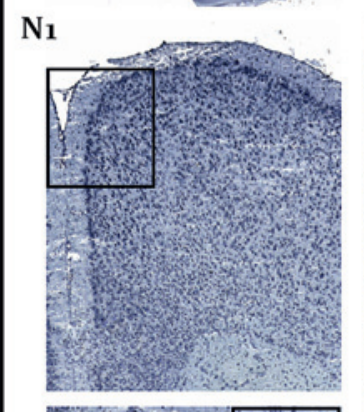
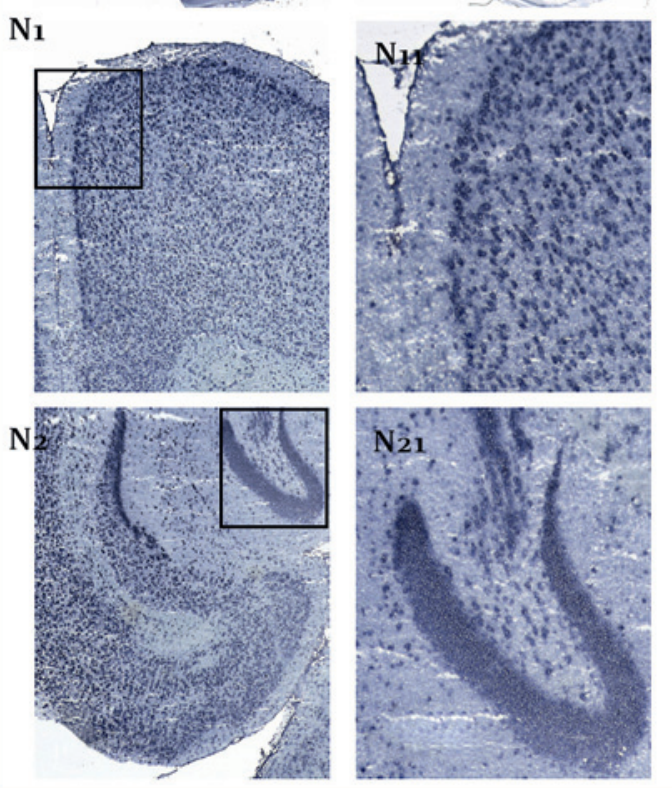

Figure 4 RNA in situ hybridisation (ISH) on E13.5 mouse embryos with a St5 riboprobe (A) in comparison with a negative control (B) showed no significant expression. On embryonic day 15.5, however, ISH with a St5 riboprobe (C) in comparison with a negative control (D) indicates that St5 is expressed in the ventricular and marginal zone of the frontal cortex (C1), as well as in the kidney (C2) and the palate (arrow) (C3). St5 is also expressed in the developing tubular structures of the kidneys of a newborn mouse (E) in comparison with a negative control (F) and of the kidneys of a 15-day-old mouse $(\mathrm{G})$ in comparison with the negative control (H). (E1) and (G1) Higher magnification of (E) and (G). (I) and (J) ISH with the St5 riboprobe (I) in comparison with neuron-specific Nissl staining (J) of a P6 mouse, demonstrating expression of St5 especially in neurons. (I1) and (J1) show the cerebellum and (I2) and (J2) the hippocampal regions under higher magnification. We also examined the brain of a 7-week-old mouse with our St5 riboprobe (K), in comparison with a negative control (L) and with Nissl staining (M), as well as the brain of an adult mouse with the St5 riboprobe (N) and a negative control (0). Signals are basically located in the cortex (N1) and in the gyrus dendatus (N2). (N11) and (N21) Higher magnification of (N1) and (N2). 
Figure 5 (A) Volume rendering of an optical projection tomography (OPT) scan of a day 9.5 St5 in situ. Expression is rendered as red at low expression levels and yellow at higher levels. The anatomy of the embryo is rendered in a greyscale colour scheme. (B) The same rendering with a sectional cut removing the right half of the embryo shows clearer detail of the expression of St5 in the neuroepithelium of the midbrain and telencephalon. (C) A sagittal section through the reconstructed day 9.5 embryo showing St5 expression in the neuroepithelium of the telencephalon, midbrain and hindbrain. (D) Volume rendering of an OPT scan of a day 11.5 embryo; ST5 expression can be seen in the developing neural region, the heart and in a regular pattern of stripes down the body axis. (E) Rendering of the signal alone allows greater appreciation of the expression in the heart (marked with an arrowed $h$ ), which seems to be in the left and right atria, and the regular striped pattern of expression (arrow). (F) A sagittal section through the reconstructed day 11.5 embryo showing St5 expression in the heart, telencephalon, midbrain and hindbrain. Expression in the heart is seen in the left atrial chamber wall (marked by an asterisk). Expression is also visible in stripes in the regions between nerves from the dorsal root ganglia. (G) An oblique view of the volume rendering highlighting expression around the lateral ventricles (arrow). (H) A coronal section showing expression in the midbrain, cerebral mesenchyme and the right and left atrial walls (marked by asterisks).
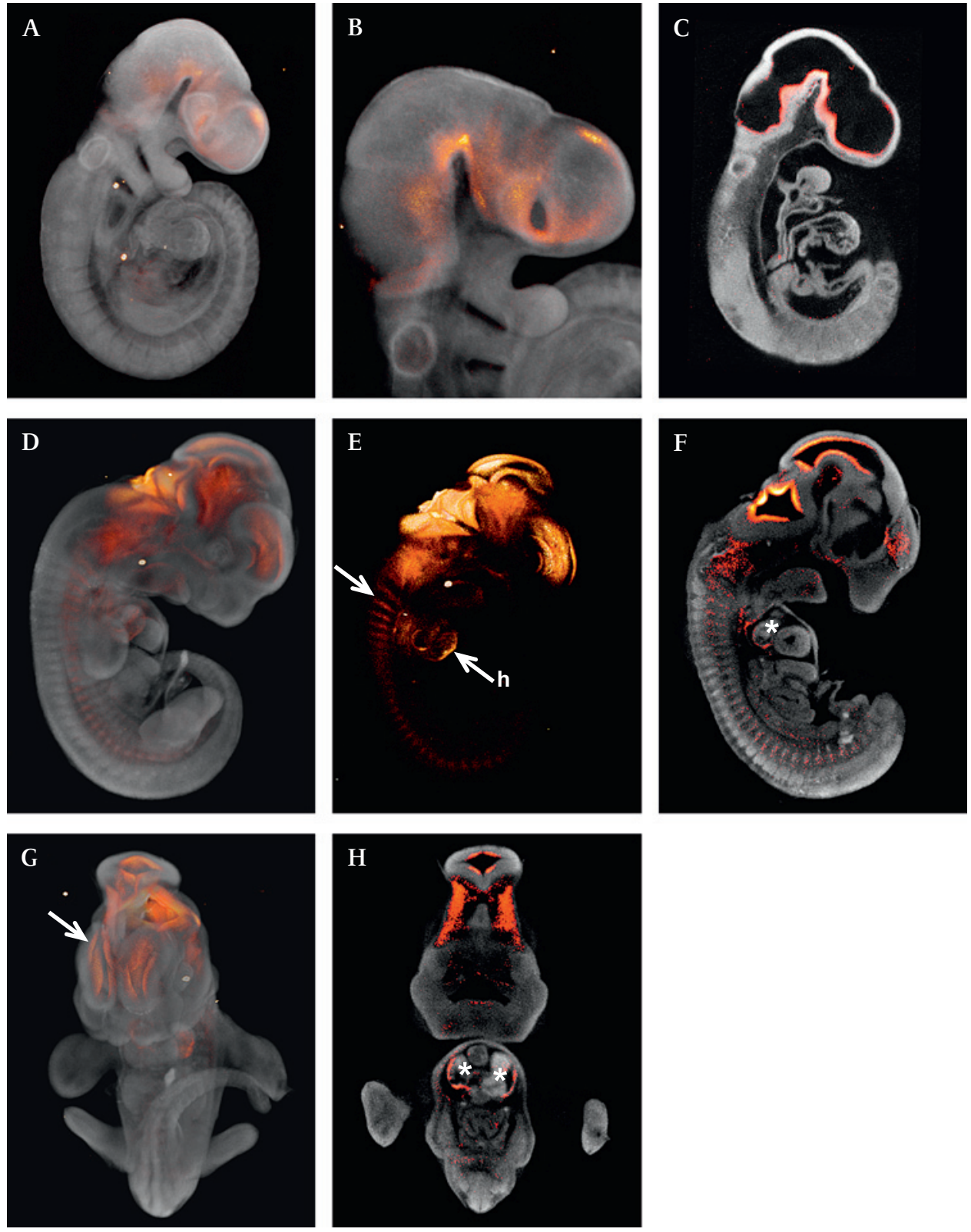

all three isoforms. Relative expression levels were compared with the mean value of ST5 expression in fetal brain. In accordance with the literature, we found that ST5 was not expressed in lymphoblasts at a relevant level. ${ }^{5}$ In RNA from human fetal tissues, we saw a relatively uniform expression pattern. In contrast, expression in most adult tissues was lower than in the corresponding fetal tissue; the highest levels were found in adult brain, kidney and skeletal muscle, with 2.5-3 times higher expression levels than in the respective fetal tissues (figure $3 \mathrm{~B}$ ).

In addition, we examined murine expression of $S t 5$ by RNA in situ hybridisation at embryonic days 8.5, 13.5 and 15 (E8.5, E13.5, E15) as well as in brain and kidneys of newborn mice on days 6, 10 and 15 (P6, P10, P15) and in brains of 7-week-old mice and adult mice. The generated riboprobe detects the mouse homologue of human exon 6, which contains the ABL1-interacting domain, and thus is specific for isoforms 1 and 3 . In accordance with the human RT-PCR studies, we found increased expression of St 5 during development (figure 4A-D and 5). Prenatally, the strongest hybridisation signals were seen in the neuroepithelium of the telencephalon, midbrain and hindbrain of an E9.5 embryo (figure 5A-C). Specific expression could also be detected in the developing neural region of the telencephalon, midbrain and hindbrain as well as in the atrial walls, and in a regular pattern of stripes in the regions between nerves from the dorsal root ganglia of an E11.5 embryo (figure 5D-H). Expression was also seen in neurons within the ventricular and marginal zone of the frontal cortex of an E15 embryo (figure 4C1) and could also be detected in various other tissues, including developing tubular structures of the kidneys and the hard palate (figure 4C2-C3, E-H). Postnatally, St 5 was expressed ubiquitously in the mouse brain, but particularly high signals were found in the cortex, hippocampal formation and cerebellum, whereas the corpus callosum showed no expression (figure $4 \mathrm{I}-\mathrm{O})$.

\section{Mutational analysis}

We had no patient with the same phenotype available for mutational analysis, but, assuming clinical variability, we performed ST5 mutational analysis in a total of 220 patients with unclassified MR, of whom 96 had moderate MR, 96 had 
severe $M R$ and 28 had $M R$ in combination with any of the further symptoms of cleft palate, deafness, kidney dysplasia or seizures. Since we found only inherited missense mutations in these patients, the ST5-related phenotype seems to be quite specific. We also sequenced ST5 in the translocation patient to exclude a mutation on the second allele.

\section{Copy-number analysis}

Neither Affymetrix 6.0 array analysis nor long-range PCR within the ST5 gene revealed any copy-number variation in the investigated control individuals.

\section{DISCUSSION}

ST5 was identified by its ability to suppress the tumorigenicity of HeLa cells in nude mice. It spans $217600 \mathrm{bp}$ and consists of four non-coding and 19 coding exons. Three isoforms of ST5 are described, all of which contain at their $3^{\prime}$ end a DENN domain (differentially expressed in neoplastic versus normal cells) region. ${ }^{67}$ Other DENN-domain-containing proteins are the Rab proteins, which belong to the Ras superfamily of GTPases. It is supposed that these domains are crucial for calcium-dependent exocytosis and therefore are closely linked to neurotransmitter release in neurons. ${ }^{8}$ Rab3GEP knock-out mice suffer from seizures and show a distinct decrease in neurotransmitter release as well as significant reduction in the number of synaptic vesicles. ${ }^{9}$ Of note, mutations in the human RABGDIA-Gen (Rab GDP dissociation inhibitor alpha) led to X-linked MR (X-linked non-specific mental retardation MRX; MIM 309541, http:// www.ncbi.nlm.nih.gov/Omim). This gene is also known to play a role in vesicle formation and transmitter release. It was also shown that DENN domains are able to activate the MAPK1/ERK2 signalling pathway which promotes cell growth and cell division. ${ }^{9}$ ST5 exon 6, which is spliced out in isoform 2, codes for an ABL1-interacting region. ${ }^{10-12}$ Therefore only isoforms 1 and 3 are capable of binding the SH3 domain of CABL1 kinase. Whereas isoform 1 stimulates the activation of the MAPK1/ERK2 pathway, isoform 3 inhibits the interaction between isoform 1 and ABL1 and thus decreases MAPK1/ERK2 activation. ${ }^{512}$ Coexpression of the two ST5 isoforms is likely to maintain the default level of ERK2 activation, as tumour cells, which lack this cell-growth-regulating function, do not express isoform $3 .^{5}$ These findings suggest that ST5 probably plays a role in cell growth and cell division, cytoskeletal organisation and tumorigenesis, as well as in formation of synaptic vesicles and exocytosis.

Disruption of the ST5 gene in our patient probably results in haploinsufficiency, but we had no suitable patient material to prove diminished expression because ST5 is not expressed in relevant levels in lymphoblasts. ${ }^{5}$ Notably, a patient with a large genomic deletion that included the ST5 gene was reported to have a phenotype similar to that of our patient, namely severe $\mathrm{MR}$, persistent hypotonia, agenesis of the corpus callosum, cleft soft palate, hyperthermic convulsions, microcephaly, facial dysmorphism and chronic otitis media. ${ }^{13}$ Furthermore, there are several recent reports showing that apparently dominant singlegene mutations and deletions in different genes are associated with severe phenotypes including MR and malformations. ${ }^{14}{ }^{15}$ In the common variation databases, one can find at least three reported variations within ST5: one $225 \mathrm{~kb}$ copy-number variation which impinges on intron 4; an 895 bp insertion and deletion (Indel) variation affecting intron 8; and a $1921 \mathrm{bp}$ Indel variation affecting intron $9 .{ }^{16} 17$ Redon et al reported the $225 \mathrm{~kb}$ copy-number variation in one of 270 control samples, whereas we have not found this aberration in 667 mentally normal controls analysed with an Affymetrix GeneChip SNP array 6.0 (unpublished data). Furthermore, we investigated 192 healthy controls by long-range PCR with reference to the published Indels affecting introns 8 and 9 and revealed neither deletion nor insertion. In addition, by mutational analysis of 220 patients with MR, we only detected a few, mostly silent, base pair exchanges. Each of the few missense variants detected were proven to be inherited from a healthy parent. Taken together, these findings provide evidence that neither the reported copynumber variations nor mutations in the ST5 gene are tolerated in the normal population, but should lead to a specific phenotypic effect.

Expression analyses by quantitative RT-PCR in fetal and adult human tissues showed ST5 to be relatively uniformly expressed in fetal tissues. ST5 expression was more pronounced in adult brain, kidney and muscle than in the corresponding fetal tissues. With RNA in situ hybridisation, we were able to show that St 5 is expressed in the frontal cortex during mouse embryonic development and in the hippocampal area and cerebellum of adult mouse brain, as well as in developing tubular structures of the kidneys and the hard palate. Thus both human and mouse expression patterns perfectly reflect the malformation pattern and brain dysfunction observed in our patient with ST5 disruption.

In summary, we suggest that ST5, like other DENN-domaincontaining Rab proteins, plays a crucial role in brain development, probably because of its assumed function in vesicle trafficking and neurotransmitter release. The latter could account not only for seizures and MR, but also for sensorineural deafness, which was also seen in our patient. The cleft palate and the kidney dysplasia in our patient, however, may be explained by other regulatory functions of ST5 in the MAPK1/ERK2 signalling pathway.

Funding This study was supported by the IZKF grant E5 to AR. MV was supported by a grant from the F.R.S.-FNRS. This work was performed as part of our research study addressing the genetics of mental retardation, which was approved by the research ethics committee of the Medical Faculty of the University of Erlangen-Nuremberg.

\section{Competing interests None.}

Patient consent Obtained.

Provenance and peer review Not commissioned; externally peer reviewed.

\section{REFERENCES}

1. Rauch A, Hoyer J, Guth S, Zweier C, Kraus C, Becker C, Zenker M, Hüffmeier U, Thiel $C$, Rüschendorf $F$, Nürnberg $P$, Reis A, Trautmann U. Diagnostic yield of various genetic approaches in patients with unexplained developmental delay or mental retardation. Am J Med Genet A 2006;140:2063-74.

2. Knight SJ, Lese CM, Precht KS, Kuc J, Ning Y, Lucas S, Regan R, Brenan M, Nicod A Lawrie NM, Cardy DL, Nguyen H, Hudson TJ, Riethman HC, Ledbetter DH, Flint J. An optimized set of human telomere clones for studying telomere integrity and architecture. Am J Hum Genet 2000;67:320-32.

3. Thiel CT, Mortier G, Kaitila I, Reis A, Rauch A. Type and level of RMRP functional impairment predicts phenotype in the cartilage hair hypoplasia-anauxetic dysplasia spectrum. Am J Hum Genet 2007:81:519-29.

4. De Gregori M, Ciccone R, Magini P, Pramparo T, Gimelli S, Messa J, Novara F, Vetro A, Rossi E, Maraschio P, Bonaglia MC, Anichini C, Ferrero GB, Silengo M, Fazzi E, Zatterale A, Fischetto R, Previderé C, Belli S, Turci A, Calabrese G, Bernardi F, Meneghelli E, Riegel M, Rocchi M, Guerneri S, Lalatta F, Zelante L, Romano C, Fichera M, Mattina T, Arrigo G, Zollino M, Giglio S, Lonardo F, Bonfante A, Ferlini A, Cifuentes F, Van Esch H, Backx L, Schinzel A, Vermeesch JR, Zuffardi 0. Cryptic deletions are a common finding in "balanced" reciprocal and complex chromosome rearrangements: a study of 59 patients. J Med Genet 2007:44:750-62

5. Hubbs AE, Majidi M, Lichy JH. Expression of an isoform of the novel signal transduction protein ST5 is linked to cell morphology. Oncogene 1999;18:2519-25.

6. Hubbard TJ, Aken BL, Beal K, Ballester B, Caccamo M, Chen Y, Clarke L, Coates G, Cunningham F, Cutts T, Down T, Dyer SC, Eyre T, Fitzgerald S, Fernandez-Banet J, Gräf S, Haider S, Hammond M, Holland R, Howe KL, Howe K, Johnson N, Jenkinson A, 
Kähäri A, Keefe D, Kokocinski F, Kulesha E, Lawson D, Longden I, Megy K, Meidl P, Overduin B, Parker A, Pritchard B, Prlic A, Rice S, Rios D, Schuster M, Sealy I, Slater G, Smedley D, Spudich G, Trevanion S, Vilella AJ, Vogel J, White S, Wood M, Birney E, Cox T, Curwen V, Durbin R, Fernandez-Suarez XM, Herrero J, Hubbard TJ, Kasprzyk A, Proctor G, Smith J, Ureta-Vidal A, Searle S. Ensembl 2007. Nucleic Acids Res 2007;35 (Database issue):D610-17.

7. Maglott D, Ostell J, Pruitt KD, Tatusova T. Entrez gene: gene-centered information at NCBI. Nucleic Acids Res 2005;33(Database issue):D54-8.

8. Yamaguchi K, Tanaka M, Mizoguchi A, Hirata Y, Ishizaki H, Kaneko K, Miyoshi J Takai Y. A GDP/GTP exchange protein for the Rab3 small G protein family up-regulates a postdocking step of synaptic exocytosis in central synapses. Proc Natl Acad Sci U S A 2002;99:14536-41.

9. Levivier E, Goud B, Souchet M, Calmels TP, Mornon JP, Callebaut I. uDENN, DENN, and dDENN: indissociable domains in Rab and MAP kinases signaling pathways. Biochem Biophys Res Commun 2001;287:688-95.

10. Lichy JH, Majidi M, Elbaum J, Tsai MM. Differential expression of the human ST5 gene in HeLa-fibroblast hybrid cell lines mediated by YY1: evidence that YY1 plays a part in tumor suppression. Nucleic Acids Res 1996;24:4700-8.

11. Majidi M, Gutkind JS, Lichy JH. Deletion of the $\mathrm{COOH}$ terminus converts the ST5 p70 protein from an inhibitor of RAS signaling to an activator with transforming activity in NIH-3T3 cells. J Biol Chem 2000;275:6560-5.

12. Majidi M, Hubbs AE, Lichy JH. Activation of extracellular signal-regulated kinase 2 by a novel Abl-binding protein, ST5. J Biol Chem 1998;273:16608-14.
13. Kleczkowska A, Fryns JP, Jaeken J, Van den Berghe H. Complex chromosomal rearrangement involving chromosomes 11, 13 and 21. Ann Genet 1988;31:126-8.

14. Mencarelli M, Spanhol-Rosseto A, Artuso R, Rondinella D, De Filippis R, BahiBuisson N, Nectoux J, Rubinsztajn R, Bienvenu T, Moncla A, Chabrol B, Villard L, Krumina Z, Armstrong J, Roche A, Pineda M, Gak E, Mari F, Ariani F, Renieri A. Nove FOXG1 mutations associated with the congenital variant of Rett syndrome. J Med Genet 2010;47:49-53.

15. Zweier C, Peippo MM, Hoyer J, Sousa S, Bottani A, Clayton-Smith J, Reardon W Saraiva J, Cabral A, Gohring I, Devriendt K, de Ravel T, Bijlsma EK, Hennekam RC, Orrico A, Cohen M, Dreweke A, Reis A, Nurnberg P, Rauch A. Haploinsufficiency of TCF4 causes syndromal mental retardation with intermittent hyperventilation (PittHopkins syndrome). Am J Hum Genet 2007;80:994-1001.

16. Mills RE, Luttig CT, Larkins CE, Beauchamp A, Tsui C, Pittard WS, Devine SE. An initia map of insertion and deletion (INDEL) variation in the human genome. Genome Res 2006:16:1182-90.

17. Redon R, Ishikawa S, Fitch KR, Feuk L, Perry GH, Andrews TD, Fiegler H, Shapero MH, Carson AR, Chen W, Cho EK, Dallaire S, Freeman JL, González JR, Gratacòs M, Huang J, Kalaitzopoulos D, Komura D, MacDonald JR, Marshall CR, Mei R, Montgomery L, Nishimura K, Okamura K, Shen F, Somerville MJ, Tchinda J, Valsesia A, Woodwark C, Yang F, Zhang J, Zerjal T, Zhang J, Armengol L, Conrad DF, Estivill X, Tyler-Smith C, Carter NP, Aburatani H, Lee C, Jones KW, Scherer SW, Hurles ME. Global variation in copy number in the human genome. Nature 2006:444:444-54. 


\section{Disruption of ST5 is associated with mental retardation and multiple congenital anomalies}

Ina Göhring, Andreas Tagariello, Sabine Endele, Claus C Stolt, Michella Ghassibé, Malcolm Fisher, Christian T Thiel, Udo Trautmann, Miikka Vikkula, Andreas Winterpacht, David R FitzPatrick and Anita Rauch

J Med Genet 2010 47: 91-98 originally published online October 19, 2009 doi: 10.1136/jmg.2009.069799

Updated information and services can be found at:

http://jmg.bmj.com/content/47/2/91

\section{These include:}

References This article cites 17 articles, 9 of which you can access for free at: http://jmg.bmj.com/content/47/2/91\#BIBL

Email alerting Receive free email alerts when new articles cite this article. Sign up in the service box at the top right corner of the online article.

Topic Articles on similar topics can be found in the following collections Collections

\section{Notes}

To request permissions go to:

http://group.bmj.com/group/rights-licensing/permissions

To order reprints go to:

http://journals.bmj.com/cgi/reprintform

To subscribe to BMJ go to:

http://group.bmj.com/subscribe/ 